\title{
Idosos vivendo com HIV - comportamento e conhecimento sobre sexualidade: revisão integrativa
}

\author{
Elderly people living with HIV - behavior and knowledge about \\ sexuality: an integrative review
}

Rosaline Bezerra Aguiar (https://orcid.org/0000-0002-4299-7035) ${ }^{1}$

Márcia Carréra Campos Leal (https://orcid.org/0000-0002-3032-7253) ${ }^{1}$

Ana Paula de Oliveira Marques (https://orcid.org/0000-0003-0731-8065) ${ }^{1}$

Kydja Milene Souza Torres (https://orcid.org/0000-0001-5258-8780) ${ }^{1}$

Maria Tereza Dantas Bezerra Tavares (https://orcid.org/0000-0002-9773-1963) ${ }^{1}$

${ }^{1}$ Centro de Ciências da

Saúde, Universidade Federal de Pernambuco. Av. Prof.

Moraes Rego 1235, Cidade

Universitária. 50670-901

Recife PE Brasil.

rosaline-rosa@hotmail.com

\begin{abstract}
The scope of this study was to identify and analyze the scientific production on behavior and knowledge about the sexuality of elderly people living with HIV. An integrative review of scientific articles indexed in the Lilacs, IBECS, Medline, BDENF, PubMed and Scopus (Elsevier) databases was conducted, considering publications from January 2007 to December 2016, using the following key words: knowledge, behavior, sexuality, Elderly, HIV/AIDS. Of the 1493 articles located, 11 were included because they met the inclusion criteria and were analyzed through two instruments: Critical Appraisal Skill Program (CASP) and Agency for Healthcare and Research and Quality (AHRQ). The data suggest that HI$V$-positive elderly people are sexually active and are involved in risk behaviors of virus transmission. It transpires that there is a limited scientific production regarding the behavior and knowledge about sexuality among elderly people living with HIV. The conclusion drawn is that the study may contribute to the improvement of public health policies that promote the approach on sexuality among elderly people, as well as the emergence of new questions regarding this issue.
\end{abstract}

Key words Knowledge, Sexuality, Elderly, HIV, Aids
Resumo O presente estudo teve como objetivo identificar e analisar a produção científica acerca do comportamento e conhecimento sobre sexualidade de idosos que vivem com HIV. Realizou-se uma revisão integrativa de artigos científicos indexados nas bases de dados, Lilacs, Ibecs, Medline, BDENF, PubMed e Scopus (Elsevier), considerando publicações a partir de janeiro de 2007 a dezembro de 2016, utilizando os seguintes descritores: conhecimento (knowledge), comportamento (behavior/behaviour) sexualidade (sexuality), idoso (Elderly), HIV/AIDS. De 1.493 artigos encontrados, 11 foram incluídos por preencherem os critérios de inclusão e foram analisados através de dois instrumentos: Critical Appraisal Skill Programme (CASP) e Agency for Healthcare and Research and Quality (AHRQ). Os dados sugerem que os idosos HIV positivo são sexualmente ativos e estão envolvidos em comportamentos de risco de transmissão do vírus. Percebe-se que existe uma produção científica limitada em relação ao comportamento e conhecimento sobre sexualidade entre os idosos que vivem com HIV. Conclui-se que o estudo poderá subsidiar políticas públicas em saúde que valorizem a abordagem sobre sexualidade na terceira idade, assim como a realização de novos questionamentos no tocante a esta temática. Palavras-chave Conhecimento, Sexualidade, Idoso, HIV, Aids 


\section{Introdução}

A sexualidade é uma das necessidades básicas do indivíduo e deve ser vivenciada em sua plenitude. Ela está presente em todas as fases da vida do ser humano. Dessa forma, a satisfação que é alcançada através do exercício da sexualidade não desaparece na velhice ${ }^{1}$. Historicamente a sexualidade dos idosos tem sido negada, entretanto, o registro crescente do número de pessoas idosas contaminadas pelo HIV mostra a necessidade de se discutir sobre esse assunto ${ }^{2}$.

Inicialmente, apenas homossexuais foram diagnosticados com HIV; em seguida, passou a ser detectado nos usuários de drogas injetáveis, nos bissexuais e heterossexuais ${ }^{3}$. No que se refere aos idosos, no começo da epidemia da aids, praticamente não foram acometidos. Nos primeiros cinco anos da epidemia no Brasil, apenas quatro casos foram diagnosticados em pessoas com 60 anos ou mais 4 .

Diferentemente, na segunda década (19902000), a aids passou a representar outro padrão epidemiológico em muitos países, inclusive no Brasil. Dessa forma, avançou entre os heterossexuais, intensificou a feminização, juvenização, interiorização, pauperização e a transmissão vertical, com progressão também entre os idosos ${ }^{5}$.

Segundo dados do Boletim Epidemiológico de 2010, a forma de transmissão predominante do HIV é por via heterossexual, tanto no sexo feminino (90,4\% dos casos) como no masculino (29,7\% dos casos). Com relação à população idosa, a infecção por HIV, até a década de 1980, tinha como principal fator causal a transmissão sanguínea; contudo, atualmente, o contato sexual é a principal causa ${ }^{6}$.

Em contrapartida, as questões sobre saúde sexual na velhice sempre tiveram baixa prioridade, tanto nas políticas públicas, quanto nas atividades e nas pesquisas, o que contribuiu para o surgimento de mitos e preconceitos em torno da sexualidade na terceira idade ${ }^{7}$. A sexualidade na velhice ainda é um assunto pouco discutido no campo da saúde, pouco percebido e entendido pela sociedade, pelos próprios idosos e pelos profissionais de saúde ${ }^{8}$.

Portanto, para se conseguir avançar na luta contra a epidemia da aids se faz necessário entender a vulnerabilidade da pessoa idosa frente a infecção pelo o HIV . Assim, após maior clareza da epidemia e de seus aspectos biológicos, epidemiológicos e psicossociais construiu-se o conceito de vulnerabilidade, que considera não só as práticas individuais, mas também características sociais, históricas e culturais, as quais acarretam maior suscetibilidade à infecção e adoecimento da população ${ }^{10}$.

O modelo ideológico que representava a aids como uma doença associada a um "grupo de risco" aumenta a vulnerabilidade individual, pois faz com que várias pessoas não se sintam suscetíveis ao HIV, desprezando a prática de sexo seguro e informações importantes sobre essa doença ${ }^{11}$. Atualmente, a aids se distribui em toda a população, e sua vulnerabilidade tornou-se condicionada a comportamentos de risco dos diversos grupos populacionais ${ }^{12}$.

O estudo dos grupos populacionais e suas vulnerabilidades são fundamentais, visto que são fatores diretamente relacionados à realidade epidemiológica atual. Assim, o grupo de homens representa a maioria dos casos de aids no país ${ }^{13,14}$; com destaque para os homens jovens, que são considerados mais vulneráveis em relação aos adultos $^{15,16}$, sendo observado o aumento de incidência de HIV entre estes indivíduos ${ }^{14,17}$.

Em relação ao grupo de mulheres, o aumento do número de casos ocorreu em todas as faixas etárias, em um processo conhecido como feminização da aids, no qual a razão entre os sexos evoluiu, passando de 14,0 homens por mulher, no ano de 1982, para 1,7 homens por mulher, em $2011^{12}$. Com relação ao grupo das pessoas idosas, que cresce com o envelhecimento da população brasileira, ocorre um aumento na incidência de casos de infecção por HIV, em detrimento à escassez de campanhas de prevenção e estudos epidemiológicos sobre sexualidade e HIV/DSTs na terceira idade ${ }^{13,18}$, somados à ampliação do período sexual ativo, processos fisiológicos do envelhecimento e aspectos comportamentais ${ }^{18}$.

Desse modo, a vulnerabilidade da pessoa idosa à infecção pelo HIV está relacionada com uma variedade de fatores, os quais colaboram para sua maior exposição ${ }^{19}$. Dentre esses fatores está o aumento da prática sexual sem preservativo e a utilização de medicamentos que melhoram e prolongam a vida sexual ${ }^{20}$. Some-se a isto, a confiança da mulher em relação ao parceiro, não exigindo o uso do preservativo, a falta de informação sobre a doença de forma geral e a carência de profissionais de saúde capacitados para perceber que o idoso está vulnerável ao HIV ${ }^{21}$.

No Brasil, o número de idosos infectados pelo HIV vem aumentando a cada ano. Dados do Ministério da Saúde (MS) mostram que, entre os anos de 1980 e 2000, o número de casos de HIV notificados em pessoas com 60 anos ou mais era de 4.761, enquanto que entre 2001 e 2016 esse número cresceu consideravelmente, chegando a 28.122 casos nessa população ${ }^{14}$. 
Este acréscimo está associado ao avanço das tecnologias de diagnóstico e assistência em HIV/ AIDS e à política brasileira de acesso universal à terapia antirretroviral (TARV). A introdução da TARV em 1996 promoveu uma melhor qualidade de vida para as pessoas infectadas com HIV e foi um marco no combate à doença ${ }^{22}$. Assim, o aumento da sobrevida causado pelo uso da TARV contribuiu para que muitas pessoas que vivem com HIV chegassem à terceira idade ${ }^{23}$.

As questões apresentadas sobre o envelhecimento associado ao diagnóstico de HIV revelam a complexidade e relevância desses eventos para a compreensão do processo saúde e doença na esfera individual, bem como no âmbito social. Implementar ações que trabalhem com a questão da sexualidade na terceira idade se tornou um desafio para as políticas públicas de saúde ${ }^{24}$.

Levando-se em consideração esses aspectos, faz-se necessário o desenvolvimento de pesquisas que investiguem o comportamento e o conhecimento sobre sexualidade em idosos que vivem com HIV, contribuindo, assim, para o desenvolvimento de políticas públicas e práticas de saúde que garantam o aperfeiçoamento do cuidado em relação à saúde sexual da população idosa, bem como a desmistificação de mitos e preconceitos, estabelecidos socialmente, acerca da sexualidade da pessoa idosa, colaborando para que esse público desfrute de uma vida sexual saudável ${ }^{25}$.

\section{Metodologia}

Para a elaboração do estudo, optou-se pelo método de revisão integrativa por ser muito utilizado na análise de conceitos, revisão de teorias ou evidências e síntese do conhecimento sobre determinado tema, permitindo identificar lacunas que precisam ser preenchidas com a realização de novos estudos. Portanto, a construção dessa revisão obedeceu as seguintes etapas: 1) elaboração da pergunta norteadora; 2) estabelecimento dos critérios de inclusão e de exclusão; 3) definição dos descritores, busca na literatura e coleta de dados; 4) análise crítica dos estudos incluídos e discussão dos resultados; e 5) apresentação da síntese do conhecimento produzido ${ }^{26,27}$.

A seleção dos artigos ocorreu no período de agosto a setembro de 2017 , sendo norteada pela seguinte pergunta: qual a produção científica acerca do comportamento e conhecimento sobre sexualidade entre os idosos que vivem com HIV?

Foram selecionados somente os artigos que atenderam aos seguintes critérios de inclusão: artigos publicados em português, espanhol e inglês, que apresentavam dados primários, qualitativos ou quantitativos, coletados entre os idosos com HIV (idade igual ou superior a 50 anos), que descreviam o comportamento ou o conhecimento em relação à sexualidade, publicados entre janeiro de 2007 e dezembro de 2016, disponíveis na Biblioteca Virtual em Saúde (BVS), a qual contempla as seguintes bases de dados relacionadas às Ciências da Saúde em Geral: Lilacs (Literatura LatinoAmericana e do Caribe em Ciências da Saúde), IBECS (Índice Bibliográfico Espanhol de Ciências da Saúde), Medline (Literatura Internacional em Ciências da Saúde), e BDENF (Base de Dados de Enfermagem). Também, buscou-se na base de dados PubMed e Scopus (Elsevier).

Foram excluídos os trabalhos que não contemplavam a temática estabelecida, publicações referentes a teses, dissertações, resumos de congresso, anais, editoriais, comentários e opiniões, artigos de revisões de literatura, estudos que incluíssem em sua amostra pessoas com idade inferior a 50 anos e artigos publicados anteriormente a 2007.

Para busca dos artigos foram utilizados os seguinte descritores indexados aos Descritores em Ciência da Saúde (DeCS) e ao Medical Subject Headings (MeSH) : conhecimento, sexualidade, idoso, comportamento, HIV e aids. Como estratégia de busca os descritores foram combinados por meio dos operadores booleanos (AND e OR).

Para avaliar a qualidade metodológica dos artigos pré-selecionados, foram aplicados dois instrumentos: Critical Appraisal Skill Programme (CASP) e Agency for Healthcare and Research and Quality (AHRQ). O CASP permite aos pesquisadores avaliar a qualidade dos estudos quantitativos e qualitativos de maneira objetiva e sistemática, além de ser de fácil operação ${ }^{26}$.

O CASP propõe um checklist que auxilia na análise crítica dos estudos quanto ao rigor, à credibilidade e à relevância por meio de 10 itens: objetivo claro e justificado; desenho metodológico apropriado aos objetivos; procedimentos metodológicos apresentados; seleção adequada da amostra; coleta de dados descrita; relação entre pesquisador e pesquisado; aspectos éticos; análise dos dados fundamentada; resultados apresentados e discutidos; importância da pesquisa ${ }^{28}$. Os estudos foram classificados segundo a pontuação obtida na aplicação do CASP, sendo: 6 a 10 pontos (boa qualidade metodológica e viés reduzido) e mínima de 5 pontos (qualidade metodológica satisfatória, porém com risco de viés aumentado $)^{29}$.

O AHRQ é uma avaliação que classifica os estudos em seis níveis de acordo com o nível de evidência: (1) revisão sistemática ou metanálise; 
(2) ensaios clínicos randomizados; (3) ensaio clínico sem randomização; (4) estudos de coorte e de caso controle; (5) revisão sistemática de estudos descritivos e qualitativos e (6) único estudo descritivo ou qualitativo ${ }^{30}$.

Para interpretação crítica dos artigos selecionados procedeu-se à análise de conteúdo ${ }^{31}$, com a apresentação da síntese do conhecimento produzido exposta por meio de discussão textual. As categorias temáticas foram construídas a partir dos conteúdos focalizados pelos estudos.

\section{Resultados}

Para a seleção dos artigos, foi realizado o cruzamento das palavras-chave, resultando o total de 1493 artigos, dos quais 52 artigos foram retirados para revisão de texto completo e apenas 11 artigos finalmente atenderam os critérios para inclusão na revisão (Figura 1). A amostra final foi representada por 11 artigos, sendo 6 nacionais e 5 internacionais. Na Tabela 1 é possível observar a seleção e exclusão dos artigos conforme cada base de dados.

Após a aplicação do CASP, foi possível constatar que os 11 artigos selecionados apresentavam uma boa qualidade metodológica e viés reduzido apresentando uma pontuação entre $6 \mathrm{a}$ 10 pontos. A tabela abaixo (Quadro 1) faz uma breve síntese das publicações que obedeceram aos critérios de inclusão.

Ao realizar a análise dos 11 artigos selecionados, foi possível observar que nos anos de 2007, 2012 e 2015 foram escritos 2 artigos por ano; nos anos de 2008, 2010, 2011, 2013 e 2016 foi encontrada uma publicação em cada ano. Não foram encontradas produções com as características dos critérios de inclusão nos de 2009 e 2014. Quanto aos países de origem, a maioria das publicações foi brasileira (54,5\%), totalizando 6 artigos, 1 artigo publicado em Uganda (9,0\%) e 4 artigos no EUA $(36,3 \%)$.

Quanto ao desenho metodológico dos artigos selecionados, a maior parte dos artigos utilizou o desenho de estudo transversal quantitativo $(81,8 \%)$, sendo 3 com características analíticas e 6 com características apenas descritivas, apresentando nível 6 na classificação da força das evidências de acordo com AHRQ. Também, foi identificado que apenas 1 artigo apresentou estudo de coorte $(9,0 \%)$, sendo classificado com nível 4 e somente 1 trabalho desenvolveu um estudo qualitativo $(9,0 \%)$, obtendo nível 6 na classificação da força das evidências.

\section{Discussão}

Os principais temas exibidos pelos artigos foram os seguintes: conhecimento sobre sexualidade na terceira idade, comportamento sexual, importância do sexo, adesão ao preservativo, consumo de drogas e os fatores que afetam os relacionamentos íntimos.

Um estudo exploratório e de natureza quantitativa realizado no Distrito Federal analisou a questão da sexualidade de pessoas idosas com diagnóstico positivo para o $\mathrm{HIV}^{32}$. Os entrevistados descreveram a atividade sexual como sendo um fator importante para manutenção da resposta sexual. $70 \%$ dos participantes relataram que o abandono da prática sexual em idosos está ligado diretamente aos fatores sociais e psicológicos. Os autores destacaram o interesse dos idosos pela sexualidade e a importância desta para sua qualidade de vida.

Por outro lado, no ambulatório da Universidade Federal de São Paulo (UNIFESP), um estudo descritivo, analítico e de corte transversal com uma amostra de 148 pessoas com 50 anos ou mais, avaliou o conhecimento e atitudes sobre sexualidade em idosos com HIV/AIDS ${ }^{33}$. Os resultados demonstraram que o conhecimento sobre a sexualidade no envelhecimento está diretamente relacionado ao sexo feminino, donas de casa, pessoas viúvas e pacientes que apresentavam comorbidades associadas ao HIV. Com relação ao comportamento, as pessoas idosas com maior escolaridade e que praticavam exercício físico foram as que apresentaram condutas mais favoráveis à sexualidade na terceira idade.

Em um estudo posterior da Universidade Federal de São Paulo (UNIFESP) foi investigada a qualidade de vida de 201 idosos com HIV relacionando ao perfil socioeconômico, conhecimento e atitudes sobre sexualidade ${ }^{34}$. Observa-se que os resultados foram semelhantes aos do estudo anterior. O conhecimento sobre a sexualidade na terceira idade foi maior entre as mulheres e os idosos desempregados, enquanto no domínio atitudes os pacientes com maior escolaridade e aqueles com idade inferior aos 60 anos foram os que demonstraram atitudes mais favoráveis à sexualidade no envelhecimento.

Também, constatou-se, através da pesquisa realizada no Hospital Presidente Vargas, em São Luís (MA), acerca do perfil comportamental de idosos com HIV/AIDS ${ }^{35}$, que a maioria dos entrevistados apresentava vida sexual ativa e que envelhecer não implicou estagnação da sexualidade. Quanto às práticas do sexo seguro, todos os idosos referiram conhecer a camisinha, no entan- 


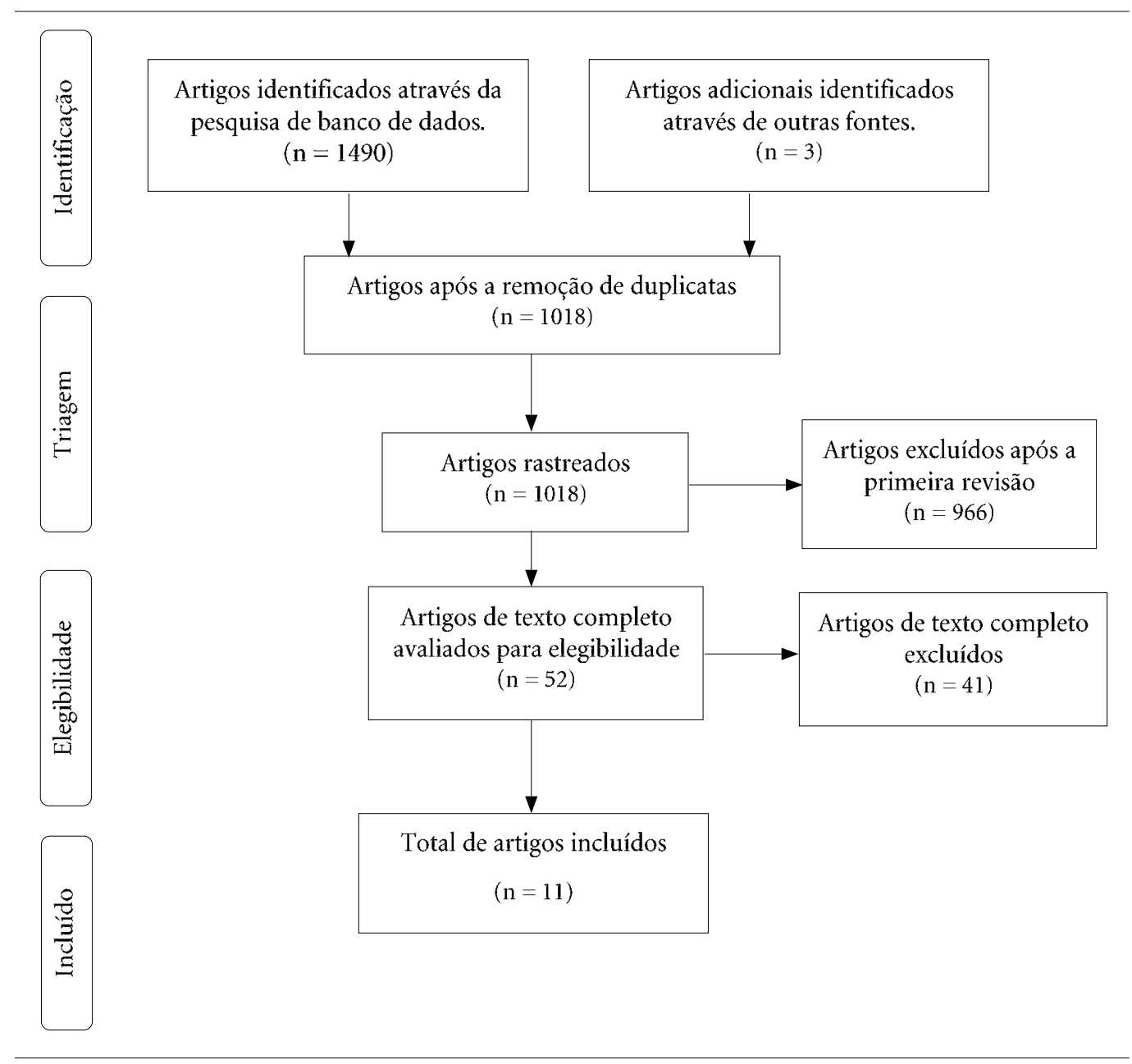

Figura 1. PRISMA Fluxograma do processo de busca e triagem dos artigos.

Fonte: Elaboração própria, 2017.

Tabela 1. Descrição dos artigos encontrados segundo base de dados.

\begin{tabular}{lcccc}
\hline \multicolumn{1}{c}{ Bases de dados } & Artigos encontrados & $\begin{array}{c}\text { Artigos pré- } \\
\text { selecionados }\end{array}$ & Artigos excluídos & Artigos inclusos \\
\hline Lilacs & 160 & 8 & 5 & 3 \\
Medline & 714 & 22 & 18 & 4 \\
Ibecs & 58 & 6 & 6 & - \\
BDENF & 73 & 4 & 2 & 2 \\
Scopus & 216 & 5 & 4 & 1 \\
PubMed & 272 & 7 & 6 & 1 \\
Total & 1493 & 52 & 41 & 11 \\
\hline
\end{tabular}

Fonte: Elaboração própria, 2017.

to, $38 \%$ mencionaram não saber usá-la e somente um pouco mais da metade da população idosa referiu usar a camisinha regularmente.

Os idosos deixam de usar o preservativo por vários motivos, como: dificuldade para utilizá-lo, pela crença da perda da ereção e da sensibilidade, à crença de que os relacionamentos afetivos ou monogâmicos conferem imunidade, porque tira o prazer e "quebra o clima"35. Esse tipo de comportamento precisa ser modificado urgentemente, pois 
Quadro 1. Distribuição dos artigos incluídos na revisão integrativa segundo título, autores, objetivos, principais resultados, ano de publicação.

\begin{tabular}{|c|c|c|c|c|c|}
\hline Título & Autores & Desenho de estudo & População & Ano & $\begin{array}{l}\text { País / } \\
\text { idioma }\end{array}$ \\
\hline $\begin{array}{l}\text { Perfil da sexualidade de } \\
\text { pessoas idosas portadoras } \\
\text { de SIDA/AIDS atendidas } \\
\text { em um serviço de saúde } \\
\text { do Distrito Federal. }\end{array}$ & $\begin{array}{l}\text { Andrade } \\
\text { PBS; } \\
\text { Benito LAO. }\end{array}$ & $\begin{array}{l}\text { Pesquisa de campo } \\
\text { exploratória, de } \\
\text { natureza quantitativa. }\end{array}$ & $\begin{array}{l}\text { Pessoas vivendo com } \\
\text { HIV, idade superior a } \\
60 \text { anos. }\end{array}$ & 2016 & $\begin{array}{l}\text { Brasil/ } \\
\text { Português }\end{array}$ \\
\hline $\begin{array}{l}\text { Qualidade de vida, } \\
\text { perfil socioeconômico, } \\
\text { conhecimento e atitude } \\
\text { sobre sexualidade de } \\
\text { "pessoas que vivem" com } \\
\text { o Vírus da Imunodeficiên- } \\
\text { cia Humana. }\end{array}$ & $\begin{array}{l}\text { Okuno, } \\
\text { MFP, et al. }\end{array}$ & $\begin{array}{l}\text { Estudo epidemiológico } \\
\text { transversal e } \\
\text { analítico, de natureza } \\
\text { quantitativa. }\end{array}$ & $\begin{array}{l}\text { Pessoas com HIV/ } \\
\text { AIDS, com idade a } \\
\text { partir de } 50 \text { anos. } \\
\text { - }\end{array}$ & 2015 & $\begin{array}{l}\text { Brasil// } \\
\text { Português }\end{array}$ \\
\hline $\begin{array}{l}\text { Sexual behavior of older } \\
\text { adults living with hiv in } \\
\text { uganda. }\end{array}$ & $\begin{array}{l}\text { Negin J, } \\
\text { et al. }\end{array}$ & $\begin{array}{l}\text { Estudo transversal } \\
\text { descritivo, quantitativo. }\end{array}$ & $\begin{array}{l}\text { Pessoas com HIV/ } \\
\text { AIDS, com idade a } \\
\text { partir de } 50 \text { anos. }\end{array}$ & 2015 & $\begin{array}{l}\text { Uganda/ } \\
\text { Inglês }\end{array}$ \\
\hline $\begin{array}{l}\text { Perfil comportamental } \\
\text { de idosos com HIV/AIDS } \\
\text { atendidos em um centro } \\
\text { de referência. }\end{array}$ & $\begin{array}{l}\text { Serra A, } \\
\text { et al. }\end{array}$ & $\begin{array}{l}\text { Estudo epidemiológico } \\
\text { transversal, descritivo, } \\
\text { de abordagem } \\
\text { quantitativa. } \\
\end{array}$ & $\begin{array}{l}\text { Idosos diagnosticados } \\
\text { com o HIV, idade } \\
\text { igual ou maior de } 60 \\
\text { anos. }\end{array}$ & 2013 & $\begin{array}{l}\text { Brasil/ } \\
\text { Português }\end{array}$ \\
\hline $\begin{array}{l}\text { Intimacy and sexual } \\
\text { decision making: exploring } \\
\text { the perspective of hiv } \\
\text { positive women over } 50 .\end{array}$ & $\begin{array}{l}\text { Psaros C, } \\
\text { et al. }\end{array}$ & Estudo qualitativo. & $\begin{array}{l}\text { Mulheres vivendo com } \\
\text { HIV, idade igual ou } \\
\text { superior a } 50 \text { anos. }\end{array}$ & 2012 & $\begin{array}{l}\text { Estados } \\
\text { Unidos/ } \\
\text { Inglês }\end{array}$ \\
\hline $\begin{array}{l}\text { Conhecimento e atitudes } \\
\text { sobre sexualidade em } \\
\text { idosos } \\
\text { portadores de HIV/AIDS. }\end{array}$ & $\begin{array}{l}\text { Okuno MFP, } \\
\text { et al. }\end{array}$ & $\begin{array}{l}\text { Estudo epidemiológico } \\
\text { de corte transversal e } \\
\text { analítico, de abordagem } \\
\text { quantitativa. } \\
\end{array}$ & $\begin{array}{l}\text { Pessoas vivendo com } \\
\text { HIV/AIDS, com idade } \\
\text { igual ou superior a } 50 \\
\text { anos. }\end{array}$ & 2012 & $\begin{array}{l}\text { Brasil/ } \\
\text { Português }\end{array}$ \\
\hline $\begin{array}{l}\text { Comportamentos em } \\
\text { saúde de uma população } \\
\text { portadora do HIV/Aids. }\end{array}$ & $\begin{array}{l}\text { Lima TC, } \\
\text { Freitas MIP. }\end{array}$ & \begin{tabular}{|l|} 
Estudo descritivo \\
exploratório, com \\
abordagem quantitativa \\
e corte transversal, \\
\end{tabular} & $\begin{array}{l}\text { Pacientes } \\
\text { soropositivos para o } \\
\text { HIV/AIDS, com } 50 \\
\text { anos ou mais. } \\
\end{array}$ & 2011 & $\begin{array}{l}\text { Brasil/ } \\
\text { Português }\end{array}$ \\
\hline $\begin{array}{l}\text { Prevalence and correlates } \\
\text { of sexual behavior and risk } \\
\text { management among hiv- } \\
\text { positive adults over } 50 .\end{array}$ & $\begin{array}{l}\text { Golub SA, } \\
\text { et al. }\end{array}$ & $\begin{array}{l}\text { Estudo analítico } \\
\text { transversal. }\end{array}$ & $\begin{array}{l}\text { Adultos vivendo com } \\
\text { HIV, com idade igual } \\
\text { ou superior a } 50 \text { anos. }\end{array}$ & 2010 & $\begin{array}{l}\text { Estados } \\
\text { Unidos/ } \\
\text { Inglês }\end{array}$ \\
\hline $\begin{array}{l}\text { Patterns and correlates } \\
\text { of sexual activity and } \\
\text { condom use } \\
\text { behavior in persons } 50 \text { - } \\
\text { plus years of age living } \\
\text { with HIV/AIDS. }\end{array}$ & $\begin{array}{l}\text { Lovejoy T, } \\
\text { et al. }\end{array}$ & $\begin{array}{l}\text { Estudo descritivo, } \\
\text { abordagem } \\
\text { quantitativa. }\end{array}$ & $\begin{array}{l}\text { Adultos infectados } \\
\text { pelo HIV com mais de } \\
50 \text { anos de idade. }\end{array}$ & 2008 & $\begin{array}{l}\text { Estados } \\
\text { Unidos/ } \\
\text { Inglês }\end{array}$ \\
\hline $\begin{array}{l}\text { Comportamento sexual } \\
\text { em adultos maiores de } 50 \\
\text { anos infectados pelo HIV. }\end{array}$ & \begin{tabular}{|l|} 
Bertoncinil \\
BZ, Moraes \\
KS, Kulkamp \\
IC. \\
\end{tabular} & $\begin{array}{l}\text { Estudo descritivo } \\
\text { transversal, abordagem } \\
\text { quantitativa. }\end{array}$ & $\begin{array}{l}\text { Adultos maiores de } \\
50 \text { anos soropositivos } \\
\text { para HIV. }\end{array}$ & 2007 & $\begin{array}{l}\text { Brasil/ } \\
\text { Português }\end{array}$ \\
\hline $\begin{array}{l}\text { Current sexual activity } \\
\text { And risky sexual behavior } \\
\text { In older men with or at } \\
\text { risk } \\
\text { For hiv infection. }\end{array}$ & $\begin{array}{l}\text { Cooperman } \\
\text { NA, Arnsten } \\
\text { JH, Klein RS. }\end{array}$ & $\begin{array}{l}\text { Estudo de coorte } \\
\text { prospectivo. }\end{array}$ & $\begin{array}{l}\text { Homens entre } 50 \text { e } 80 \\
\text { anos, com ou em risco } \\
\text { de infecção pelo HIV. }\end{array}$ & 2007 & $\begin{array}{l}\text { Estados } \\
\text { Unidos/ } \\
\text { Inglês }\end{array}$ \\
\hline
\end{tabular}

Fonte: Elaboração própria, 2017. 
a prática do sexo desprotegido dissemina o HIV, aumentando a contaminação nesta faixa etária.

Durante um estudo descritivo exploratório de corte transversal sobre comportamentos de idosos com HIV/AIDS, realizado na cidade de Campinas, no interior do Estado de São Paulo ${ }^{36}$, os pesquisadores verificaram que o número de homens sexualmente ativos era maior do que o de mulheres. A análise do comportamento de saúde identificou que poucos faziam uso de bebida alcoólica $(12,8 \%)$, cigarro $(3,7 \%)$ e drogas para estimulação sexual $(2,8 \%)$. Os autores concluíram que a população estudada era sexualmente ativa e que passou a utilizar preservativo nas relações sexuais após o diagnóstico do HIV.

Em Santa Catarina foi desenvolvido um estudo a respeito do comportamento sexual em adultos maiores de 50 anos infectados pelo $\mathrm{HIV}^{37}$, no qual os autores verificaram que 95,5\% dos entrevistados referiram vida sexualmente ativa. Apesar de conhecerem e já terem usado preservativo, $50 \%$ dos idosos relataram não usá-lo regularmente independente da soropositividade do parceiro. Esse comportamento é muito preocupante, pois mesmo sabendo que são soropositivos e que podem transmitir o vírus, estes indivíduos praticam sexo sem proteção ${ }^{37}$.

Em Uganda, um estudo transversal e descritivo investigou o comportamento sexual de homens $(n=42)$ e mulheres $(n=59)$ vivendo com HIV com mais de 50 anos em que foi constatado que apenas $14 \%$ das mulheres relataram ser sexualmente ativas em comparação com $49 \%$ dos homens ${ }^{38}$. Foi observado que a maior parte dos idosos sexualmente ativos não usava preservativo. Nenhuma mulher com mais de 60 anos relatou ser sexualmente ativa, embora homens com mais de 60 anos tenham referido atividade sexual semelhante aos homens mais jovens.

Para a população masculina a atividade sexual foi vista como sendo muito importante, enquanto que para as mulheres foi menos importante. Os autores sugerem que este achado pode explicar, em parte, o menor número de atividade sexual relatada pela população idosa feminina em comparação com os indivíduos idosos do sexo masculino. Enquanto 30\% dos participantes declararam fazer uso de álcool, apenas 10\% revelaram ter feito sexo sob influência de bebidas alcoólicas ${ }^{38}$.

Os Resultados obtidos através de uma pesquisa em Nova York (EUA), que examinou a prevalência e correlação do comportamento sexual, risco sexual e estratégias de redução do risco comportamental em adultos vivendo com HIV, com idade igual ou superior a $50 \operatorname{anos}^{39}$, demonstraram que a maioria dos idosos apresentava vida sexual ativa, com uma frequência de atividade sexual entre 2 a 3 vezes por semana. $49 \%$ dos participantes sexualmente ativos afirmaram fazer usos de preservativo como estratégia de gerenciamento de risco.

Observa-se que $45 \%$ dos participantes relataram uso de drogas (álcool, maconha, estimulantes), destes, 36\% referiram usar durante o sexo. Os homens homossexuais/bissexuais foram os mais propensos a reportar o uso de drogas durante atividade sexual. As mulheres foram as que menos apresentaram esse tipo de comportamento. $\mathrm{O}$ consumo de substâncias psicoativas apresentou associação significativa com o comportamento sexual de risco, pois as pessoas idosas que mencionaram estar sob efeito de drogas durante o ato sexual tiveram mais que o dobro de chances de praticar sexo sem proteção ${ }^{39}$.

A intoxicação por uso de drogas é conhecida por prejudicar o julgamento, aumentar a impulsividade e dificultar a capacidade de negociar interações sociais, favorecendo, assim, a prática de relações sexuais sem preservativo ${ }^{40}$. Essas descobertas enfatizam a importância de avaliar e abordar o uso de drogas entre os indivíduos idosos que vivem com $\mathrm{HIV}^{39}$.

Ademais, em três áreas metropolitanas do leste e meio-oeste dos Estados Unidos (EUA) um estudo examinou os comportamentos sexuais em idosos infectados pelo HIV com mais de 50 anos de idade $^{41}$. Dos participantes, $38 \%$ foram sexualmente ativos nos últimos três meses, sendo a atividade sexual mais comum em homens heterossexuais $(72 \%)$. Uma porcentagem muito menor de homens homossexuais/bissexuais (36\%) e mulheres heterossexuais $(21 \%)$ relatou atividade sexual. Entre as pessoas sexualmente ativas, apenas $27 \%$ dos homens heterossexuais relataram uso irregular de preservativo em comparação com $37 \%$ dos homens gays/bissexuais e 35\% das mulheres heterossexuais.

Igualmente, um estudo transversal investigou a frequência de atividade sexual e os fatores associados ao comportamento sexual de risco entre 624 idosos, com ou em risco de infecção pelo $\mathrm{HIV}^{40}$. Os pesquisadores descobriram que apenas $18 \%$ dos homens HIV negativos e 58\% dos homens HIV positivos usaram sempre preservativos com seus parceiros sexuais. Os fatores associados ao comportamento sexual de risco incluíram falta de infecção pelo HIV, uso de drogas e maior importância do sexo.

Já em Boston nos Estados Unidos, um estudo qualitativo realizado com 19 mulheres com idade superior a 50 anos (idade média $=56,79$ ), que vivem com HIV, identificou o que as mulheres idosas pensam a respeito dos relacionamentos ín- 
timos $^{42}$. A maioria das idosas expressou o desejo de ter uma relação íntima com um parceiro, mas descreveu múltiplos desafios para prosseguir com um relacionamento. No geral, as mulheres descreveram uma sensação de desesperança acerca de estarem em um relacionamento íntimo. Este sentimento parecia ser alimentado pelo estigma, preocupações sobre a imagem corporal e os dilemas levantados pela divulgação da soropositividade para o HIV.

O público feminino relatou que, por receio das reações dos parceiros, optavam por absterse de relacionamentos íntimos em vez de lidar com as possíveis repercussões da divulgação da infecção pelo HIV. As idosas sentiam que as mudanças que ocorriam em seus corpos, agravadas pelos efeitos da TARV, as tornavam sexualmente indesejáveis para um parceiro. As relações amorosas são amplamente vistas como determinantes importantes da qualidade de vida, assim pessoas idosas com HIV precisam de apoio para lidar com as preocupações relacionadas à imagem corporal e gerenciar a divulgação do diagnóstico para o parceiro íntimo ${ }^{42}$.

Os trabalhos incluídos nesta revisão foram realizados em contextos socioculturais diversos, abrangendo estudos nacionais e internacionais, qualitativos, quantitativos e uma avaliação de intervenção. Entretanto, apesar das altas taxas de prevalência do HIV em muitos países da África subsaariana, onde a transmissão sexual é o modo dominante de infecção pelo HIV, apenas uma pesquisa acerca do comportamento sexual de idosos com HIV foi encontrada nesta região do continente africano.

À medida que a população de idosos que vivem com HIV se torna cada vez mais vulnerável e em crescimento, as ações de prevenção primária e secundárias de redução de risco são urgentemente necessárias para ajudar a mitigar a propagação do HIV $^{39}$. Nesse sentido, a sensibilização dos profissionais da área da saúde com relação a vulnerabilidade do idoso ao HIV é muito importante, para que as questões da sexualidade na velhice sejam levadas em conta. Os achados deste estudo sugerem que as ações de prevenção às IST/HIV/ AIDS não só devem ser adaptadas à idade, mas também ao gênero e orientação sexual com o intuito de reduzir efetivamente o comportamento sexual de risco entre a população idosa.

\section{Conclusão}

Os dados sugerem que os idosos HIV positivo são sexualmente ativos e estão envolvidos em com- portamentos de risco de transmissão do vírus. $\mathrm{O}$ número de homens com mais de um(a) parceiro(a) sexual e com uma vida sexualmente ativa é maior do que o de mulheres. Para a população masculina, a atividade sexual foi vista como sendo muito importante, sendo que estes foram os que mais apresentaram comportamento sexual de risco, demonstrando bastante resistência quanto ao uso do preservativo.

O consumo de substâncias psicoativas e o fator "maior importância do sexo" apresentou associação significativa com o comportamento sexual de risco. Esta pesquisa mostrou que os idosos que vivem com HIV enfrentam barreiras significativas para manterem relações sexuais saudáveis enquanto envelhecem, o que reforça a continuidade de comportamentos sexuais de risco.

Dessa forma, é importante entender que a percepção de risco é diferente entre os grupos de pessoas em suas diversas faixas etárias, motivadas pelos aspectos socioeconômicos, demográficos e culturais aos quais estão expostos. Logo, se faz necessário considerar o processo de adoecimento de forma coletiva, compreendendo melhor como os determinantes sociais contribuem para a disseminação da doença.

A partir desta revisão foi possível observar que a produção científica em relação ao comportamento e conhecimento sobre sexualidade entre os idosos que vivem com HIV ainda é muito reduzida, o que ressalta a necessidade de pesquisas no tocante a esta temática para subsidiar e estimular o desenvolvimento de políticas públicas que trabalhem a questão da saúde sexual na terceira idade, com intuito de promover a participação destes idosos em práticas sexuais mais seguras, tanto para manter sua própria saúde como para prevenir a propagação do vírus para outras pessoas.

\section{Colaboradores}

RB Aguiar participou na concepção e delineamento do estudo, análise e interpretação dos dados, redação do artigo e aprovação final do manuscrito. MCC Leal participou do delineamento e orientação da pesquisa, revisão crítica do texto e aprovação final do manuscrito. APO Marques participou da revisão crítica relevante do conteúdo intelectual e aprovação final da versão a ser publicada. KMS Torres e MTDB Tavares participaram da redação e aprovação da versão final do manuscrito. 


\section{Referências}

1. Vallescar D. Sexualidade na Terceira Idade. 2006. [acessado 2017 Nov 22]. Disponível em: http://www. webartigos.com/articles/11942/1/sexualidade-na-terceira-idade/paginal.html

2. Zornitta M. Os novos idosos com aids e desigualdade à luz da bioética [dissertação]. Rio de Janeiro: Escola Nacional de Saúde Pública Sergio Arouca; 2008.

3. Brasil. Ministério da Saúde (MS). Guia de Vigilância Epidemiológica. $7^{a}$ ed. Brasília: MS; 2009. caderno 6: aids, Hepatites virais, Sífilis congênita, Sífilis em gestantes. p. 1-22. [acessado 2017 Out 20]. Disponível em: http://bvsms.saude.gov.br/bvs/publicacoes/guia vigilancia_epidemiologica_7ed.pdf.

4. Godoy VS, Ferreira MD, Silva EC, Gir E, Canini SRMS. O Perfil epidemiológico da Aids em idosos utilizando Sistemas de Informação em Saúde do DATASUS: realidades e desafios. J Bras Doenças Sexualmente Transmissiveis 2008; 20(1). [acessado 2017 Set 20]. Disponível em: http://www.dst.uff.br/revista20-1-2008/1.pdf

5. Caldas JMP, Gessolo KM. AIDS depois dos 50: um novo desafio para as políticas de saúde pública. In: VII congresso virtual de HIV/AIDS. O VIH/SIDA na criança e no idoso; 2007. Anais. p. 127-131. [acessado 2017 Out 09] Disponível em: http://www.associacaoamigosdagrandeidade.com/wpcontent/uploads/filebase/consultoria/LIVRO\%20VII\%20CONGRESSO $\% 20$ VIRTUAL $\% 20$ HIV \%20AIDS\%20o \%20hiv $\% 20$ aids $\% 20$ na $\% 20$ crian $\%$ C3\%83\%C2\%A7a $\% 20$ e $\% 20$ no\%20idoso.pdf

6. Brasil. Ministério da Saúde (MS). Atenção à saúde da pessoa idosa e envelhecimento. Brasília: MS; 2010. (Série B. Textos Básicos de Saúde). (Série Pactos pela Saúde 2006, v. 12). [acessado 2017 Set 17]. Disponível em: http://bvsms.saude.gov.br/bvs/publicacoes/atencao_saude_pessoa_idosa_envelhecimento_v12.pdf

7. Gott M. Sexual health and the new ageing. Age and Ageing. Oxford Journal Medicine \& Health Age and Ageing 2006; 35(2). [acessado 2017 Nov 15]. Disponível em: http://ageing.oxfordjournals.org/content/35/2/106.full

8. Oliveira DC, Oliveira EG, Gomes AMT, Teotônio MC, Wolter RMCP. O significado do HIV/AIDS no processo do envelhecimento. Revista de Enfermagem UERJ 2011; 19(3). [acessado 2017 Nov 15]. Disponível em: https://www.yumpu.com/pt/document/ view/40173348/o-significado-do-hiv-aids-no-processo-de-envelhecimento

9. Saldanha AA, Araújo LF. A aids na terceira idade na perspectiva dos idosos, cuidadores e profissionais de saúde. In: Congresso-Comunicação-Tema: Clínica e Tratamento. $7^{\circ}$ Congresso Virtual HIV/AIDS -10 out. 2006. [acessado 2017 Nov 17]. Disponível em: http:// www.aidscongresso.net/7congresso.php

10. Ayres JRCM, França Junior I, Calazans GJ, Saletti Filho HC. O conceito de vulnerabilidade e as práticas de saúde: novas perspectivas e desafios. In: Czeresnia $\mathrm{D}$, Freitas CM, organizadores. Promoção da saúde: conceitos, reflexões, tendências. Rio de Janeiro: Fiocruz: 2009. p. 117-139.
11. Paulio MAS, Jeolás LS. Aids, drogas, riscos e significados: uma construção sociocultural. Cien Saude Colet 2005; 10(1):175-184. [acessado 2017 Nov 15]. Disponível em: http://www.scielo.br/scielo.php?script=sci_arttext\&pid $=$ S1413-81232005000100024\&lng=pt \&nrm=iso

12. Brasil. Ministério da Saúde (MS). Boletim Epidemiológico HIV - AIDS-DST. Brasília: MS; 2012. [acessado 2017 Nov 20]. Disponível em: http://bvsms.saude.gov. br/bvs/periodicos/boletim_epidem_hivaids_2012. pdf.

13. Nakamura AS, Tanaka MA, Bonafé SM. Aids e infecção pelo hiv no brasil: uma revisão bibliográfica dos grupos populacionais. 2013. Anais Eletrônico -VIIIIII EPCC - Encontro Internacional de Produção Científica Cesumar UNICESUMAR - Centro Universitário Cesumar. Editora CESUMAR, Maringá - Paraná Brasil, 2013. [acessado 2017 Set 25]. Disponível em: http://www.cesumar.br/prppge/pesquisa/epcc2013/ oit_mostra/amanda_sayuri_nakamura.pdf

14. Brasil. Ministério da Saúde (MS). Boletim epidemiológico AIDS-DST 2016. ano V; no 1. [acessado 2017 Set 18]. Disponível em: http://bvsms.saude.gov.br/bvs/ periodicos/boletim_epidem_hivaids_2012.pdfhttp:// www.aids.govbr/sites/default/files/anexos/publicacao/2016/59291/boletim_2016_1_pdf_16375.pdf

15. Guerriero I, Ayres JRCM , Hearst M. Masculinidade e vulnerabilidade ao HIV de homens heterossexuais, São Paulo, SP. Rev Saude Publica 2002; 36(Supl. 4):5060.

16. Villarinho L, Bezerra I, Lacerda R, Latorre MRDO, Paiva V, Stall R, Hearst N. Caminhoneiros de rota curta e sua vulnerabilidade ao HIV. Rev Saude Publica 2002; 36(4):61-67. [acessado 2017 Nov 26] Disponível em: http://www.sidastudi.org/resources/inmagic-img /dd2246.pdf

17. Rios LF. Parcerias e práticas sexuais de jovens homossexuais no Rio de Janeiro. Cad Saude Publica 2003; 19(Supl. 2):S223-S232. [acessado 2017 Nov 28]. Disponível em: https://pdfs.semanticscholar.org/62d7/47b04fee4c74a78d316be16a5c56f91ea223.pdf

18. Dornelas Neto J, Nakamura A, Cortez LER, Yamaguchi MU. Doenças sexualmente transmissíveis em idosos: uma revisão sistemática. Cien Saude Colet 2015; 20(12):3853-3864. [acessado 2017 Out 21]. Disponível em: http://www.scielo.br/pdf/csc/v20n12/14138123-csc-20-12-3853.pdf

19. Delmiro RS. O que pensam os idosos sobre a AIDS representações sociais e Práticas [dissertação]. Universidade Estadual do Sudoeste da Bahia; 2011. [acessado 2017 Set 22]. Disponível em: <http://www.uesb. br/ppgenfsaude/dissertacoes/turma1/Disserta $\%$ C3\%A7\%C3\%A3o\%20Rosana\%20Delmiro.pdf>.

20. Andrade HAS, Silva SK, Santos MIPO. Aids em idosos: vivências dos doentes. Escola Anna Nery 2010; 14(4). [acessado 2017 Out 21]. Disponível em: http://www. scielo.br/scielo.php?script=sci_arttext\&pid $=$ S1414$81452010000400009 \& \operatorname{lng}=$ en\&nrm $=$ iso\&tlng $=$ pt

21. Silva CM, Lopes FMVM, Vargens OMC. A vulnerabilidade da mulher idosa em relação à Aids. Revista Gaúcha de Enfermagem 2010; 31(3). [acessado 2017 Out 11]. Disponível em: http://www.scielo.br/pdf/ rgenf/v31n3/v31n3a07.pdf 
22. Beck C. Análise de Sobrevida e perfil epidemiológico de casos de AIDS em Porto Alegre/RS: limitações e potencialidades da vigilância epidemiológica [dissertação]. Porto Alegre: Universidade Federal do Rio Grande do Sul; 2014. [acessado 2017Out 16]. Disponível em: file://C:/Users/rosAline/Downloads/analise $\% 20$ de\%20sobrevida\%20idoso\%20HIV.pdf

23. Brasil. Ministério da Saúde (MS). Envelhecimento e saúde da pessoa idosa. Brasília: MS; 2006. [acessado 2017 Out 11]. Disponível em: http://dab.saude.gov. br/portaldab/biblioteca.php?conteudo=publicacoes/ cab19

24. Melo HMA, Leal MCC, Marques APO, Marino JG. O conhecimento sobre aids de homens idosos e adultos jovens: um estudo sobre a percepção desta doença. Cien Saude Colet 2012; 17(1):43-53.

25. Cerqueira MBR, Rodrigues RN. Fatores associados à vulnerabilidade de idosos vivendo com HIV/AIDS em Belo Horizonte (MG), Brasil. Cien Saude Colet 2016; 21(11):3331-3338

26. Cunha PLP, Cunha CS, Alves PF. Manual de revisão bibliográfica sistemática integrativa: a pesquisa baseada em evidências. Belo Horizonte: Ed. Ănima Educação; 2014. [acessado 2017 Out 16]. Disponível em: http:// disciplinas.nucleoead.com.br/pdf/anima_tcc/gerais/ manuais/manual_revisao.pdf

27. Mendes KDS, Silveira RCCP, Galvão CM. Revisão Integrativa: método de pesquisa para incorporação de evidências na saúde e na Enfermagem. 2008. [acessado 2017 Out 16]; 17(4):758-764. Disponível em: http://www.scielo.br/scielo.php?script=sci_arttext\&pid $=$ S0104-07072008000400018

28. Alencar DL. Fatores associados ao exercício da sexualidade de pessoas idosas [dissertação]. Recife: Universidade Federal de Pernambuco; 2013.

29. Toledo MM, Takahashi RF, De-La-Torre-Ugarte-Guanilo MC. Elementos de vulnerabilidade individual de adolescentes ao HIV/AIDS. Rev Bras Enferm 2011; 6(2):370-375. [acessado 2017 Out 17]. Disponível em: http://www.scielo.br/pdf/reben/v64n2/a24v64n2.pdf

30. Stillwell S, Melnyk BM, Fineout-Overholt E, Williamson K. Evidence-based practice: step by step. Am J Nurs 2010; 110(5):41-47. [acessado 2017 Nov 25]. Disponível em: http://journalEs.lww.com/ajnonline/ Fulltext/2010/01000/Evidence_Based_Practice_. Step_by_Step__The_Seven.30.aspx

31. Bardin L. L'Analyse de contenu. Paris: Presses Universitaires de France; 1977.

32. Andrade PBS, Benito LAO. Perfil da sexualidade de pessoas idosas portadoras de SIDA/AIDS atendidas em um serviço de saúde do Distrito Federal. Universitas 2016; 14(2):105-113.

33. Okuno MFP, Fram DS, Batista REA, Barbosa DA, Belasco AGS. Conhecimento e atitudes sobre sexualidade em idosos portadores de HIV/AIDS. Acta Paulista Enfer 2012; 25(1):115-121.

34. Okuno MFP, Gosuen GC, Campanharo CRV, Fram DS, Batista REA, Belasco AGS. Qualidade de vida, perfil socioeconômico, conhecimento e atitude sobre sexualidade de "pessoas que vivem" com o Vírus da Imunodeficiência Humana. Rev. Latino-Am. Enfermagem 2015; 23(2):192-199.
35. Serra A, Sardinha AHL, Lima SCVS, Pereira ANS. Perfil comportamental de idosos com hiv/aids atendidos em um centro de referência. Rev Enferm UFPE 2013; 7(2):407-413. [acessado 2017 Out 20]. Disponível em: http://www.repositorio.ufc.br/bitstream/ riufc/7586/1/2013_art_acpjcosta.pdf

36. Lima TC, Freitas MIP. Comportamentos em saúde de uma população portadora do HIV/Aids. Rev Bras Enferm 2012; 65(1):110-115. [acessado 2017 Out 20]. Disponível em: http://www.scielo.br/scielo.php?scrip$\mathrm{t}=$ sci_arttext\&pid=S0034-71672012000100016

37. Bertoncini BZ, Moraes KS, Kulkamp IC. Comportamento sexual em adultos maiores de 50 anos infectados pelo hiv. DST - J Bras Doenças Sex Transm 2007; 19(2):75-79. [acessado 2017 Out 20]. Disponível em: http://www.dst.uff.br/revista19-2-2007/3.pdf.

38. Negin J, Geddes L, Brennan-Ing M, Kuteesa M, Karpiak S, Seeley J. Sexual Behavior of Older Adults Living with HIV in Uganda. Archives of Sexual Behavior 2016; 45(2):441-449.

39. Golub SA, Tomassilli CJ, Pantalone DW, Brennan M, Karpiak SE, Parsons JT. Prevalence and Correlates of Sexual Behavior and Risk Management Among HIV -Positive Adults Over 50. Sexually Transmitted Diseases 2010; 37(10). [acessado 2017 Out 27]. Disponível em: http://www.natap.org/2010/HIV/CorrelatesSexual.pdf

40. Lovejoy TI, Heckman TG, Sikkema KJ, Hansen NB, Kochman A, Suhr JA, Garske JP, Johnson CJ. Patterns and Correlates of Sexual Activity and Condom Use Behavior in Persons 50-Plus Years of Age Living with HIV/AIDS. AIDS Behav 2008; 12(6):943-956. [acessado 2017 Out 27]. Disponível em: https://www.ncbi. nlm.nih.gov/pmc/articles/PMC2575000/.

41. Cooperman NA, Arnsten JH, Klein RS. Current sexual activity and risky sexual behavior in older men with or at risk for hiv infection. AIDS Education and Prevention 2007; 19(4):321-333.

42. Psaros C, Barinas J, Robbins GK, Bedoya A, Safren SA, Park ER. Intimacy and sexual decision making: Exploring the perspective of HIV positive women over 50 . AIDS Patient Care and STDs 2012; 26(12):755-760.

Artigo apresentado em 26/11/2017

Aprovado em 25/06/2018

Versão final apresentada em 27/06/2018 